\title{
Nachwuchspreis der DeGEval 2020
}

\section{Edith Halves}

Ziel des Preises ist es, jährlich eine Nachwuchsevaluatorin/einen Nachwuchsevaluator oder auch eine Nachwuchsgruppe auszuzeichnen, um so eine herausragende Arbeit im Bereich Evaluation im deutschsprachigen Raum zu würdigen. Aus Sicht der DeGEval soll dieser Preis sowohl die Bedeutung des wissenschaftlichen Nachwuchses auf dem Gebiet der Evaluation insgesamt hervorheben als auch der Nachwuchsförderung dienen.

Für den Nachwuchspreis 2020 wurden insgesamt drei Beiträge eingereicht: zwei Qualifikationsarbeiten (Masterarbeit) und eine Praxisarbeit. Neben den unterschiedlichen Formaten wurden unterschiedliche Themenfelder behandelt. Dies stellte die Jury, der neben der Autorin auch Patrica Berndt, Christian Erzberger, Jessica Prigge, Thomas Widmer und Angela Wroblewski angehörten, bei ihrer Entscheidung vor einige Herausforderungen. Schließlich hat sich die Jury für die Masterarbeit von Julia Nick entschieden.

Bei dem ausgewählten Wettbewerbsbeitrag handelt es sich um eine Abschlussarbeit im Rahmen des Masterstudiengangs Evaluation der Universität des Saarlandes und der Hochschule für Technik und Wirtschaft des Saarlandes.

Der Titel der Arbeit lautet: Chancen und Grenzen der Evaluation informeller Bildungsprozesse von Kindern und Jugendlichen. Eine Evaluationssynthese mit besonderem Fokus auf der Begleitforschung des Projektes „Lernpaten Saar“.

Die Arbeit überzeugt durch die Aktualität des Themas, das eine Forschungslücke schließt. Gleichzeitig wird es anwendungsbezogen anhand des Projektes „Lernpaten Saar" bearbeitet. Informelles Lernen als bedeutsamer Aspekt Lebenslangen Lernens erfährt in einer Vielzahl von Politikfeldern, Projekten und Programmen Aufmerksamkeit, so dass das Thema für den gesamten Bereich der Evaluation von hoher Relevanz und Bedeutung sowie international anschlussfähig ist.

Frau Nick hat sich der Herausforderung gestellt, einen solchen Gegenstand ein aus Sicht von Evaluierenden überaus ,schlecht strukturierter" und entsprechend „schlecht zu strukturierender“ Gegenstand, weil er diffus Verwendung findet - zu evaluieren. Es ist ihr aus Sicht der Jury hervorragend gelungen, dies in stringenter und nachvollziehbarer Weise in ihrer Arbeit darzulegen. Die Autorin stellt den Forschungsstand zum Thema umfassend dar und zeigt die Relevanz des Themas auf.

Inhaltlich geht die Arbeit über den Rahmen einer Masterarbeit hinaus - gerade auch, weil die Erkenntnisse der Evaluation einer übergeordneten Reflexion von The- 
orie und Praxis unterzogen werden und sie so die Grundlage für weitere Evaluationen in diesem Bereich bildet. Dies und die Rückbindung der Evaluation an die theoretischen Implikationen des Untersuchungsgenstandes machen die Besonderheiten der Arbeit aus. Gerade bei der Frage, wie etwas evaluiert werden kann, das eigentlich nicht „messbar“ ist, zeigt die Arbeit ihre Stärke. Die Grenzen von Evaluationen in dem Bereich informellen Lernens werden plausibel dargestellt. Die methodischen Beschreibungen sind gut nachvollziehbar und sind dem Evaluationsgegenstand angemessen. Eine leicht kritische Anmerkung sei gestattet: Ein Verweis auf die DeGEval-Standards hätte die Arbeit bereichern können.

Der Evaluationsgegenstand selbst ist von erheblicher Bedeutung, gerade in dem hier betrachteten Bereich der Kinder- und Jugendarbeit, aber auch als Unterstützung bei Maßnahmen der Hilfen zur Erziehung - im Sinne weit übergreifender Prozesse, die nicht direkt an klassische Lernorte gebunden sind (z.B. Sportvereine).

Somit geht der diesjährige 14. DeGEval-Nachwuchspreis an Frau Julia Nick. Wir gratulieren herzlich!

Wir freuen uns, auch in diesem Jahr den Preis wieder an eine Masterarbeit vergeben zu können und hoffen damit auch weiterhin Studierende zur Einreichung ihrer Arbeit für den nächstjährigen DeGEval-Nachwuchspreis zu motivieren.

Die Jury des Nachwuchspreises der DeGEval e.V. 2020 